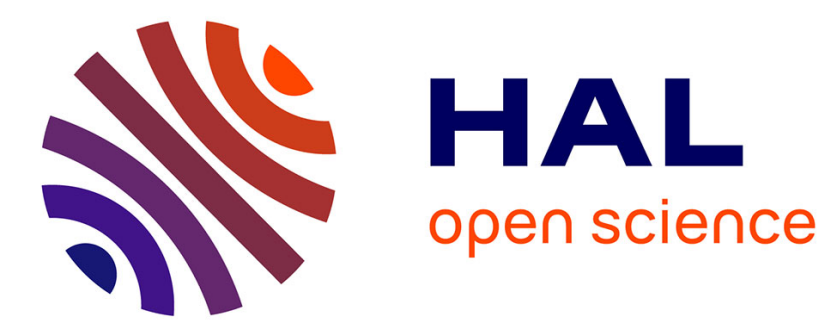

\title{
Sur l'écart quadratique moyen des diviseurs d'un entier normal, 2
}

Gérald Tenenbaum

\section{To cite this version:}

Gérald Tenenbaum. Sur l'écart quadratique moyen des diviseurs d'un entier normal, 2. Mathematical Proceedings of the Cambridge Philosophical Society, 2005, 138, pp.1-8. hal-00091189

\section{HAL Id: hal-00091189 \\ https://hal.science/hal-00091189}

Submitted on 5 Sep 2006

HAL is a multi-disciplinary open access archive for the deposit and dissemination of scientific research documents, whether they are published or not. The documents may come from teaching and research institutions in France or abroad, or from public or private research centers.
L'archive ouverte pluridisciplinaire HAL, est destinée au dépôt et à la diffusion de documents scientifiques de niveau recherche, publiés ou non, émanant des établissements d'enseignement et de recherche français ou étrangers, des laboratoires publics ou privés. 
Math. Proc. Camb. Phil. Soc.

38 (2005), 1-8.

\title{
Sur l'écart quadratique moyen des diviseurs d'un entier normal, 2
}

\author{
PAR GÉrald Tenenbaum \\ Institut Élie Cartan, Université Henri Poincaré-Nancy 1, \\ BP 239, 54506 Vandouvre Cedex, France
}

\section{Introduction et énoncé des résultats}

Considérons la fonction arithmétique

$$
D^{*}(n, t):=\sum_{\substack{d\left|n, d^{\prime}\right| n \\ 0<\left|\log \left(d^{\prime} / d\right)\right| \leqslant t}} 1 \quad(n>1, t>0),
$$

qui généralise la fonction $T^{*}(n, \alpha):=D^{*}\left(n,(\log n)^{-\alpha}\right)$, étudiée dans la première partie de cet article [7]. Les variations de $D^{*}(n, t)$ au voisinage de l'origine permettent de mesurer, en moyenne quadratique, la propension des diviseurs de l'entier $n$ à s'agglutiner autour de certaines valeurs. Nous nous proposons ici de donner une majoration essentiellement optimale de cette fonction de $t$ sur $[0,1]$ lorsque $n$ est astreint à parcourir un ensemble d'entiers non exceptionnels en un certain sens quantitativement précisé.

Conformément à l'usage, nous désignons dans ce travail par $\tau(n)$ le nombre des diviseurs, et par $\Omega(n)$ le nombre des facteurs premiers comptés avec multiplicité, d'un entier naturel $n$.

Théorème 1.1. Soit $\beta<\beta_{0}:=(\log 2) / \log \{2 /(\mathrm{e}-1)\} \approx 4,5655$. Il existe des constantes positives $\varrho=\varrho(\beta)$ et $C=C(\beta)$ telles que, pour tous $z>1, t \in] 0,1]$, l'inégalité

$$
D^{*}(n, t) / 2^{\Omega(n)} \leqslant z t^{\beta}
$$

ait lieu pour tous les entiers $n \leqslant x$ sauf au plus $C x(t / z)^{\varrho}$ exceptions.

La constante $\beta_{0}$ de cet énoncé est optimale : pour

$$
0<\alpha \leqslant(1-1 / \mathrm{e}) \log \{2 /(\mathrm{e}-1)\} \approx 0,09597, \quad x \rightarrow \infty, \quad t:=(\log x)^{-\alpha},
$$

le théorème 1 de [7] fournit

$$
D^{*}(n, t)=2^{\Omega(n)} t^{\beta_{0}+o(1)}
$$

pour $x+o(x)$ entiers $n \leqslant x$.

En choisissant $z=z(x)$ tendant vers l'infini arbitrairement lentement et, par exemple, $t=1 / z, \beta=2$, dans le Théorème 1.1 nous obtenons un nouveau renseignement de nature quadratique sur la proximité des diviseurs de presque tout entier. Ici et dans la suite, nous convenons de désigner par pp (presque partout) une relation satisfaite pour tous les entiers d'une suite de densité naturelle unité.

Corollaire 1.2. La relation

$$
\sum_{\substack{d\left|n, d^{\prime}\right| n \\\left|\log \left(d^{\prime} / d\right)\right|<\varepsilon(n)}} 1=o(\tau(n)) \quad \mathrm{pp}
$$

est valable pour toute fonction $\varepsilon(n)$ qui tend vers 0 lorsque $n$ tend vers l'infini. 
Le Théorème 1.1 permet également de contrôler les petits rapports de diviseurs consécutifs d'un entier normal. Désignons par $\left\{d_{j}(n)\right\}_{j=1}^{\tau(n)}$ la suite croissante des diviseurs d'un entier $n$ et posons, pour $\alpha \geqslant 0, n>1$,

$$
B(n, \alpha):=\sum_{1 \leqslant j<\tau(n)}\left\{\log \left(d_{j+1}(n) / d_{j}(n)\right)\right\}^{-\alpha} .
$$

Cette fonction mesure en moyenne la taille des petits rapports $d_{j+1}(n) / d_{j}(n)$. Nous obtenons le résultat suivant où nous notons $\overline{\mathbf{d}} \mathcal{A}$ la densité naturelle supérieure d'une suite d'entiers $\mathcal{A}$.

Théorème 1.3. Pour tout $\alpha \in\left[0, \beta_{0}[\right.$, la fonction arithmétique $B(n, \alpha) / \tau(n)$ admet une loi de répartition limite. De plus, il existe une constante $c(\alpha)>0$ telle que

$$
\overline{\mathbf{d}}\{n: B(n, \alpha)>z \tau(n)\} \ll 1 / z^{c(\alpha)} \quad(z>1) .
$$

Nous appliquons ensuite ce résultat à l'étude de la fonction

$$
\tau(n, \vartheta):=\sum_{d \mid n} d^{i \vartheta}
$$

qui est, à une normalisation près, la transformée de Fourier-Stieltjes de la fonction de répartition des $\log d$ pour $d \mid n$. Hall a montré dans [2] que l'on a pour tout $\vartheta \neq 0$ fixé

$$
|\tau(n, \vartheta)|<\xi(n) \sqrt{\log _{2} n}
$$

et, dans $[5]$ (§3.2), Hall et Tenenbaum ont soulevé la question de déterminer, pour $a>0$, $b>0$, la borne inférieure $\lambda(a, b)$ de l'ensemble des nombres réels $\lambda$ tel que

$$
\max _{a \leqslant \vartheta \leqslant a+b}|\tau(n, \vartheta)|<\tau(n)^{\lambda} \quad \text { pp. }
$$

Dans cette perspective, Hall [4] a établi que, pour tout $\alpha>0$ et tout intervalle $I(n)$ de longueur $|I(n)|:=(\log n)^{\alpha}$, on a

$$
\frac{1}{|I(n)|} \int_{I(n)}|\tau(n, \vartheta)|^{2} \mathrm{~d} \vartheta \sim \tau(n) \quad \text { pp. }
$$

Nous obtenons ici une forme «finie» de cette formule.

Théorème 1.4. Il existe une constante $c>0$ telle que l'on ait pour $z>1$

$$
\left.\sup _{a, b \in \mathbb{R}}\left|\int_{a}^{a+b}\right| \tau(n, \vartheta)\right|^{2} \mathrm{~d} \vartheta-b \tau(n) \mid \leqslant z \tau(n)
$$

pour tous les entiers $n>1$ sauf au plus ceux d'une suite de densité supérieure $\ll 1 / z^{c}$.

Ce résultat est une conséquence immédiate du Théorème 1.3, au vu d'une formule générale de Montgomery et Vaughan (voir [6], formule (28) p. 140) relative aux polynômes de Dirichlet et qui implique, dans le cas de $\tau(n, \vartheta)$,

$$
\left.\left|\int_{a}^{a+b}\right| \tau(n, \vartheta)\right|^{2} \mathrm{~d} \vartheta-b \tau(n) \mid \leqslant 3 B(n, 1) .
$$

Nous déduisons ensuite du Théorème 1.4 une extension de $(1 \cdot 3)$ et une minoration de $\lambda(a, b)$. 
Corollaire 1.5. Soient $L(n)$ une quantité tendant vers l'infini avec $n$ et $I(n)$ un intervalle arbitraire de longueur $L(n)$. Alors on a

$$
\frac{1}{L(n)} \int_{I(n)}|\tau(n, \vartheta)|^{2} \mathrm{~d} \vartheta \sim \tau(n) \quad \text { pp. }
$$

Corollaire 1.6. Il existe un nombre réel $b_{0}>0$ tel que

$$
\inf _{a>0, b \geqslant b_{0}} \lambda(a, b) \geqslant \frac{1}{2} .
$$

Corollaire 1.7. Soit $\lambda^{*}(b):=\sup _{a>0} \lambda(a, b)$. Alors on $\operatorname{ainf}_{b>0} \lambda^{*}(b) \geqslant \frac{1}{2}$.

Ce dernier résultat découle du précédent en remarquant que

$$
\lambda^{*}\left(b_{1}+b_{2}\right) \leqslant \max \left\{\lambda^{*}\left(b_{1}\right), \lambda^{*}\left(b_{2}\right)\right\} \quad\left(b_{1}>0, b_{2}>0\right) .
$$

Posons $F(u):=2^{u} \Gamma\left(\frac{1}{2}+\frac{1}{2} u\right) /\left\{\sqrt{\pi} \Gamma\left(1+\frac{1}{2} u\right)\right\}$. Il est à noter, dans ce contexte, que Hall a établi dans [3] la majoration

$$
\sup _{a>0, b>0} \lambda(a, b) \leqslant \frac{F^{\prime}(v)}{F(v) \log 2} \approx 0,94119
$$

où $v$ est l'unique solution de l'équation $v F^{\prime}(v) / F(v)=1+\log F(v)$.

L'auteur tient à exprimer ici ses plus chaleureux remerciements à Michel Balazard pour ses questions et suggestions, à l'origine de ce travail.

\section{Démonstration du Théorème 1.1}

Nous utiliserons à plusieurs reprises le lemme 50.1 de [5], relatif à la fonction

$$
\Omega(n, w):=\sum_{p^{\nu} \| n, p \leqslant w} \nu
$$

et qui stipule que, pour chaque $\varepsilon \in] 0, \frac{1}{2}[$, on a

$$
\sup _{w \geqslant 3}\left\{\Omega(n, w)-(1+\varepsilon) \log _{2} w\right\} \leqslant T
$$

sauf pour au plus $\ll(1+\varepsilon)^{-T} \varepsilon^{-2} x$ entiers $n \leqslant x$.

Nous posons

$$
\nabla(n ; t, y):=\sum_{\substack{d d^{\prime}\left|n,\left(d, d^{\prime}\right)=1 \\ 0<\right| \log \left(d^{\prime} / d\right) \mid \leqslant t}} y^{\Omega\left(d d^{\prime}\right)} .
$$

Le résultat suivant complète le théorème 4 de [7] qui fournit une majoration de $\nabla(n ; t, y)$ pour $\frac{1}{2}(\mathrm{e}-1) \leqslant y \leqslant 1, t>0$, lorsque $n$ est «normal . 
Lemme 2.1. Soient $0<y<\frac{1}{2}(\mathrm{e}-1), z>1,0<t \leqslant 1, x \geqslant 2$. Il existe un nombre réel $\kappa=\kappa(y)>0$ tel que l'on ait

$$
\nabla(n ; t, y) \leqslant z t \log (1+1 / t)
$$

pour tous les entiers $n \leqslant x$ sauf au plus $\ll_{y} x / z^{\kappa}$.

Démonstration. Nous employons la méthode utilisée pour établir le théorème 50 de [5]. Comme les détails techniques sont très voisins, nous nous contentons ici d'indications relativement succinctes.

Observons d'abord que nous pouvons restreindre l'étude au cas $t>x^{-1 / 10}$ : en effet, dans la circonstance opposée, la majoration triviale

$$
\sum_{n \leqslant x} \nabla(n ; t, y) \leqslant 2 \sum_{1 \leqslant d^{\prime} \leqslant d \mathrm{e}^{t}} \frac{x}{d d^{\prime}} \ll x t \log x \asymp x t \log (1 / t) \quad(x \geqslant 2)
$$

permet immédiatement de conclure.

Supposons donc, dans toute la suite de cette démonstration, que $x$ est assez grand et que $x^{-1 / 10}<t \leqslant 1$. Nous avons, pour toutes valeurs considérées des variables,

$$
\nabla(n ; t ; y) \leqslant 2 \sum_{1<d \leqslant \mathrm{e}^{t}} y^{\Omega(d)}+2 \sum_{1<d<d^{\prime} \leqslant \mathrm{e}^{t} d} y^{\Omega\left(d d^{\prime}\right)} .
$$

La première somme est vide si $t<\log 2$ et n'excède pas 1 dans le cas contraire. Nous pouvons donc nous borner à estimer la seconde lorsque, disons, $x^{-1 / 10}<t \leqslant \frac{1}{2}$. Pour tout couple $\left(d, d^{\prime}\right)$ compté dans cette sommation, nous avons

$$
\mathrm{e}^{-t} / d<1 / d^{\prime}<\log \left\{d^{\prime} /\left(d^{\prime}-1\right)\right\} \leqslant \log \left(d^{\prime} / d\right) \leqslant t,
$$

donc $d \geqslant d_{0}(t):=\max \left(1, \mathrm{e}^{-t} / t\right)$. Posons

$$
\left.\left.\left.\left.I_{1}:=\left[d_{0}(t), 1 / t^{2}\right], \quad I_{2}:=\right] 1 / t^{2}, x^{1 / 4}\right], \quad I_{3}:=\right] x^{1 / 4}, \sqrt{x}\right],
$$

et désignons par $\nabla_{j}(n)$ la contribution à la somme double de $(2 \cdot 3)$ des couples $\left(d, d^{\prime}\right)$ tels que $d \in I_{j}$. Nous avons d'abord, en majorant $y$ par 1 dans $\nabla_{1}(n)$,

$$
\sum_{n \leqslant x} \nabla_{1}(n) \leqslant \sum_{d_{0}(t) \leqslant d \leqslant 1 / t^{2}} \sum_{d<d^{\prime} \leqslant d \mathrm{de}^{t}} \frac{x}{d d^{\prime}} \ll x t \log (1 / t) .
$$

Cela implique que $\nabla_{1}(n) \leqslant z t \log (1 / t)$ sauf peut-être pour $\ll x / z$ entiers $n$ exceptionnels.

Soient $\varepsilon, v$ des paramètres satisfaisant à $0<\varepsilon<\frac{1}{2}, 0<v<1$. En appliquant $(2 \cdot 1)$ avec $T:=\frac{1}{2}(\log z) /|\log v|$, nous obtenons que, pour $j=2$ ou 3, l'inégalité

$$
\nabla_{j}(n) \leqslant \sqrt{z} \nabla_{j}^{*}(n)
$$

a lieu pour tous les entiers $n \leqslant x$ sauf au plus $\ll x /\left(\varepsilon^{2} z^{\kappa}\right)$ avec

$$
\kappa=\kappa(\varepsilon, v):=\frac{1}{2} \log (1+\varepsilon) /|\log v|
$$

et

$$
\nabla_{j}^{*}(n):=\sum_{\substack{d \mid n \\ d \in I_{j}}} \sum_{\substack{d^{\prime} \mid n / d \\ d<d^{\prime} \leqslant d \mathrm{e}^{t}}} v^{\Omega\left(n, d^{\prime}\right)}\left(\log d^{\prime}\right)^{-(1+\varepsilon) \log v} y^{\Omega\left(d d^{\prime}\right)}
$$


Nous choisirons plus loin la valeur des paramètres $\varepsilon$ et $v$ en fonction de $y$.

Considérons d'abord le cas $j=2$. Nous pouvons écrire

$$
\begin{aligned}
S_{2}^{*}: & =\sum_{n \leqslant x} \nabla_{2}^{*}(n) \\
& =\sum_{d \in I_{2}} \sum_{d<d^{\prime} \leqslant d \mathrm{e}^{t}}(y v)^{\Omega\left(d d^{\prime}\right)}\left(\log d^{\prime}\right)^{-(1+\varepsilon) \log v} \sum_{n \leqslant x / d d^{\prime}} v^{\Omega\left(n, d^{\prime}\right)} .
\end{aligned}
$$

Pour tout couple $\left(d, d^{\prime}\right)$ apparaissant dans cette sommation, on a $d d^{\prime} \leqslant \mathrm{e}^{t} d^{2} \leqslant 2 \sqrt{x}$. La somme intérieure est donc

$$
\ll \frac{x}{d d^{\prime}}\left(\log d^{\prime}\right)^{v-1}
$$

Il suit

$$
S_{2}^{*} \ll x \sum_{d \in I_{2}} \frac{(y v)^{\Omega(d)}}{d} \sum_{d<d^{\prime} \leqslant d \mathrm{e}^{t}}(y v)^{\Omega\left(d^{\prime}\right)} \frac{\left(\log d^{\prime}\right)^{v-1-(1+\varepsilon) \log v}}{d^{\prime}} .
$$

Comme $\left(\mathrm{e}^{t}-1\right) d>t d>\sqrt{d}$ pour tout $d \in I_{2}$, nous pouvons majorer la somme intérieure par sommation d'Abel en faisant appel au théorème de Shiu [8]. Posant

$$
A=A(\varepsilon, y, v):=v(y+1)-2-(1+\varepsilon) \log v,
$$

nous obtenons que cette somme intérieure est $\ll t(\log d)^{A}$. En reportant dans $(2 \cdot 5)$, il suit

$$
S_{2}^{*} \ll x t \sum_{d} \frac{(y v)^{\Omega(d)}(\log d)^{A}}{d} \ll x t \sum_{d} \frac{(\log d)^{A+y v-1}}{d} .
$$

Nous choisissons $v=(1+\varepsilon) /(2 y+1)$, de sorte que, notant $Q(z):=z \log z-z+1 \geqslant 0$,

$$
A+y v-1=(1+\varepsilon) \log (2 y+1)-2-Q(1+\varepsilon) .
$$

Si $y<\frac{1}{2}(\mathrm{e}-1)$, cette expression est $<-1$ pour $\varepsilon=\varepsilon(y)>0$ assez petit. D'où

$$
S_{2}^{*} \ll t x
$$

Compte tenu de $(2 \cdot 4)$, cela implique $\nabla_{2}(n) \leqslant z t$ sauf pour au plus $\ll x / \sqrt{z}$ entiers $n \leqslant x$.

Procédons de manière semblable pour majorer $S_{3}^{*}:=\sum_{n \leqslant x} \nabla_{3}^{*}(n)$. Nous obtenons d'abord

$$
S_{3}^{*} \ll \frac{x}{(\log x)^{(1+\varepsilon) \log v}} \sum_{d \in I_{3}} \frac{(y v)^{\Omega(d)}}{d} \sum_{d<d^{\prime} \leqslant \min \left(\mathrm{e}^{t} d, x / d\right)} \frac{(y v)^{\Omega\left(d^{\prime}\right)}}{d^{\prime}}\left(\log \frac{2 x}{d d^{\prime}}\right)^{v-1} .
$$

Comme $t>x^{-1 / 10}$, nous pouvons de nouveau faire appel au théorème de Shiu pour estimer la somme intérieure : elle est

$$
\ll t(\log d)^{y v-1}\left(\log \frac{2 x}{d^{2}}\right)^{v-1} .
$$

En reportant dans $(2 \cdot 6)$, nous parvenons finalement à

$$
S_{3}^{*} \ll t x(\log x)^{(1+\varepsilon) \log (2 y+1)-2-Q(1+\varepsilon)} \ll t x,
$$


pour le choix $v=(1+\varepsilon) /(2 y+1)$ avec $y<\frac{1}{2}(\mathrm{e}-1)$ et $\varepsilon=\varepsilon(y)$ convenablement choisi dans $] 0, \frac{1}{2}\left[\right.$. Nous concluons comme précédemment que $\nabla_{3}(n) \leqslant z t$ avec au plus $\ll x / \sqrt{z}$ exceptions.

En regroupant nos estimations, nous obtenons bien que $(2 \cdot 2)$ est valable avec la majoration annoncée pour le nombre d'exceptions.

Nous sommes maintenant en mesure de compléter la démonstration du Théorème 1.1.

Nous avons

$$
D^{*}(n, t)=\sum_{\begin{array}{c}
d d^{\prime} \mid n,\left(d, d^{\prime}\right)=1 \\
0<\left|\log \left(d^{\prime} / d\right)\right| \leqslant t
\end{array}} \tau\left(n / d d^{\prime}\right)
$$

d'où

$$
\tau(n) \max _{1 \leqslant k \leqslant \Omega(n)} V_{k}^{*}(n, t) / 2^{k} \leqslant D^{*}(n, t) \leqslant 2^{\Omega(n)} \sum_{1 \leqslant k \leqslant \Omega(n)} V_{k}^{*}(n, t) / 2^{k},
$$

avec

$$
V_{k}^{*}(n, t):=\sum_{\begin{array}{c}
d d^{\prime} \mid n,\left(d, d^{\prime}\right)=1 \\
\left.0<\mid \log \left(d^{\prime}\right) d\right) \mid \leqslant t \\
\Omega\left(d d^{\prime}\right) \leqslant k
\end{array}} 1 .
$$

Soit $\beta \in] 0, \beta_{0}\left[\right.$. Lorsque $t^{4} z>1$, nous nous contentons d'insérer l'inégalité

$$
V_{k}^{*}(n, t) \leqslant \nabla(n ; t, y) / y^{k} \quad(k \geqslant 0)
$$

dans la majoration de (2.7). Grâce au Lemme 2.1, nous obtenons que l'on a, pour $\frac{1}{2}<y<\frac{1}{2}(\mathrm{e}-1), Z \gg 1$,

$$
\frac{D^{*}(n ; t)}{2^{\Omega(n)}} \leqslant \frac{2 y}{2 y-1} \nabla(n ; t, y) \leqslant Z t \log (1+1 / t)
$$

pour tous les entiers $n \leqslant x$ sauf au plus $\ll_{y} x / Z^{\kappa}$. Le résultat annoncé en découle en choisissant $Z:=z t^{\beta-1} / \log (1+1 / t)$ : le nombre des entiers exceptionnels est alors

$$
\ll_{y} \frac{x\{\log (1+1 / t)\}^{\kappa}}{z^{\kappa} t^{(\beta-1) \kappa}} \ll x(t / z)^{\kappa(1-4 \beta / 19)} .
$$

Lorsque $t^{4} z \leqslant 1$, nous déduisons de $(2 \cdot 8)$ et du Lemme 2.1 que, pour $\frac{1}{2}<y<\frac{1}{2}(\mathrm{e}-1)$, $W \gg 1$,

$$
V_{k}^{*}(n, t) \leqslant W t y^{-k} \log (1+1 / t) \quad(k \geqslant 0)
$$

pour tous les entiers $n \leqslant x$ sauf au plus $\ll_{y} x / W^{\kappa}$. Donnons-nous un paramètre $\left.\varepsilon \in\right] 0,1[$ et choisissons

$$
W:=\frac{z^{\varepsilon / 4}}{t^{\varepsilon} \log (1+1 / t)} \gg(z / t)^{\varepsilon / 4} \gg 1 .
$$

Nous obtenons que

$$
V_{k}^{*}(n, t) \leqslant z^{\varepsilon / 4} t^{1-\varepsilon} y^{-k} \quad(k \geqslant 0)
$$

pour tous les entiers $n \leqslant x$ sauf au plus $\ll x(t / z)^{\kappa \varepsilon / 4}$. Comme $V_{k}^{*}(n, t)$ est entier, cela implique en particulier que $V_{k}^{*}(n, t)=0$ pour $0 \leqslant k<k_{0}$ avec

$$
k_{0}:=\left[\frac{\log \left(z^{\varepsilon / 4} t^{-(1-\varepsilon)}\right)}{|\log y|}\right] \geqslant\left[\frac{\log z}{4|\log y|}\right],
$$


et donc $t^{-(1-\varepsilon)} z^{-\varepsilon / 4} \leqslant y^{k_{0}}<t^{-(1-\varepsilon)} z^{-\varepsilon / 4} y^{-1}$. Pour les entiers $n$ non exceptionnels, nous avons donc, en vertu de $(2 \cdot 7)$,

$$
\frac{D^{*}(n, t)}{2^{\Omega(n)}} \leqslant \sum_{k \geqslant k_{0}} \frac{z^{\varepsilon / 4} t^{1-\varepsilon}}{(2 y)^{k}} \leqslant \frac{2 y z^{\varepsilon / 4} t^{1-\varepsilon}}{(2 y-1)(2 y)^{k_{0}}} \leqslant \frac{2 y}{(2 y-1) 2^{k_{0}}} \leqslant \frac{4 y t^{u}}{2 y-1}
$$

avec $u:=(1-\varepsilon)(\log 2) /|\log y|$. Choisissons $\varepsilon$ et $y$ de sorte que $u>\beta$. Il existe alors un $t_{0}=t_{0}(\varepsilon, y)$ tel que, pour tout $t \leqslant t_{0}$, le majorant de $(2 \cdot 9)$ n'excède pas $t^{\beta}$. Nous obtenons donc bien que, pour $t \leqslant t_{0}, z \leqslant t^{-1 / 4}$, le nombre des entiers $n \leqslant x$ contrevenant à $(1 \cdot 1)$ est $\ll_{\beta} x(t / z) \varrho$ avec $\varrho=\varepsilon \kappa / 4$. Comme le résultat est trivialement vérifié lorsque $t_{0}<t \leqslant 1, z \leqslant t^{-1 / 4}$, cela termine la démonstration.

\section{Démonstration du Théorème 1.3}

Lemme 3.1. Soit $0<\alpha<\beta_{0}$. Il existe $\kappa=\kappa(\alpha)>0$ tel que, pour tout $z>1$, on ait

$$
\overline{\mathbf{d}}\{n>1: B(n, \alpha) \leqslant z \tau(n)\} \ll 1 / z^{\kappa} .
$$

Démonstration. On a clairement

$$
\begin{aligned}
B(n, \alpha) & \leqslant \sum_{\substack{d\left|n, d^{\prime}\right| n \\
0<\left|\log \left(d^{\prime} / d\right)\right| \leqslant 1}}\left\{\frac{1}{\left|\log \left(d^{\prime} / d\right)\right|^{\alpha}}-1\right\}+T(n, 0) \\
& =J(n)+T(n, 0),
\end{aligned}
$$

avec $J(n):=\alpha \int_{0}^{1} D^{*}(n, t) \mathrm{d} t / t^{\alpha+1}$ et $T(n, 0):=D^{*}(n, 1)+\tau(n)$. Comme $T(n, 0) \leqslant z \tau(n)$ sauf au plus pour les entiers $n$ d'une suite de densité $\ll(\log 2 z) / z$ (cf. [5], chapitre 4$)$, on voit qu'il suffit d'établir l'assertion de l'énoncé avec $J(n)$ à la place de $B(n, \alpha)$. En scindant l'intervalle d'intégration en intervalles dyadiques, nous pouvons écrire

$$
J(n) \leqslant \sum_{k \geqslant 0} D^{*}\left(n, 2^{-k}\right) 2^{\alpha(k+1)} .
$$

D'après le Théorème 1.1 , on $\mathrm{a}$, pour tout $\beta<\beta_{0}$,

$$
\sup _{k \geqslant 0} D^{*}\left(n, 2^{-k}\right) 2^{\beta k} \leqslant z 2^{\Omega(n)}
$$

sauf peut-être pour les entiers $n$ d'une suite de densité supérieure

$$
\ll_{\beta} \sum_{k \geqslant 0} \frac{1}{2^{\varrho k} z^{\varrho}} \ll_{\beta} \frac{1}{z^{\varrho}} .
$$

Choisissons, par exemple, $\beta=\left(\alpha+\beta_{0}\right) / 2$, et posons

$$
K:=2^{\alpha} \sum_{k \geqslant 0} 2^{-\left(\beta_{0}-\alpha\right) k / 2}=\frac{2^{\left(\beta_{0}+\alpha\right) / 2}}{2^{\left(\beta_{0}-\alpha\right) / 2}-1} .
$$

Nous obtenons que $J(n) \leqslant K z 2^{\Omega(n)}$ pour tous les entiers $n$ sauf peut-être ceux d'une suite de densité supérieure $\ll_{\alpha} 1 / z^{\varrho}$. Comme $\left\{2^{\Omega(n)} / \tau(n)\right\}^{1 / 2}$ est de valeur moyenne bornée, cela implique bien le résultat annoncé. 
Le Théorème 1.3 est une conséquence facile du Lemme 3.1.

Pour $\varepsilon>0$, désignons par $B_{\varepsilon}(n, \alpha)$ la sous-somme de $B(n, \alpha)$ restreinte aux indices $j$ tels que $\log \left\{d_{j+1}(n) / d_{j}(n)\right\}>\varepsilon$. D'après le théorème 1 de $[1], B_{\varepsilon}(n, \alpha) / \tau(n)$ possède une loi de répartition limite $F_{\varepsilon}$. Bien entendu, $F_{\varepsilon}$ est une fonction croissante de $\varepsilon$.

Soit $\delta \in] 0, \beta_{0}-\alpha[$. Alors

$$
0 \leqslant B(n, \alpha)-B_{\varepsilon}(n, \alpha) \leqslant \varepsilon^{\delta} B(n, \alpha+\delta) \quad(\varepsilon>0, n>1)
$$

donc, d'après le Lemme 3.1, il existe une constante positive $\kappa=\kappa(\alpha, \delta)>0$ telle que la densité supérieure de l'ensemble des entiers $n$ satisfaisant à

$$
B(n, \alpha)-B_{\varepsilon}(n, \alpha)>\varepsilon^{\delta / 2} \tau(n)
$$

soit $\ll_{\alpha, \delta} \varepsilon^{\kappa \delta / 2}$. Cela implique, lorsque $x \rightarrow \infty$,

$$
F_{\varepsilon}\left(z-\varepsilon^{\delta / 2}\right)+O\left(\varepsilon^{\kappa \delta / 2}\right)+o(1) \leqslant \frac{1}{x} \sum_{\substack{n \leqslant x \\ B(n, \alpha) \leqslant z \tau(n)}} 1 \leqslant F_{\varepsilon}(z)+o(1) .
$$

On en déduit le résultat annoncé en faisant tendre $x$ vers l'infini puis $\varepsilon$ vers 0 .

\section{Bibliographie}

[1] P. Erdős \& G. Tenenbaum, Sur les diviseurs consécutifs d'un entier, Bull. Soc. Math. de France 111 (1983), 125-145.

[2] R.R. Hall, Sums of imaginary powers of the divisors of integers, J. London math. Soc. (2) 9 (1975), $571-580$.

[3] R.R. Hall, Uniform upper bounds for the complex divisor function, Math. Proc. Camb. Phil. Soc. 108 (1990), 421-427.

[4] R.R. Hall, $\Omega$ theorems for the complex divisor function, Math. Proc. Camb. Phil. Soc. 115 (1994), $145-157$.

[5] R.R. Hall \& G. Tenenbaum, Divisors, Cambridge tracts in mathematics 90, Cambridge University Press (1988).

[6] H.L. Montgomery, Ten lectures on the interface between analytic number theory and harmonic analysis, CBMS regional conferences series in mathematics, no. 84, American mathematical society, Providence, Rhode Island (1994), 220 pp.

[7] A. Raouj \& G. Tenenbaum, Sur l'écart quadratique moyen des diviseurs d'un entier normal, Math. Proc. Camb. Phil. Soc. 126 (1999), 399-415.

[8] P. Shiu, A Brun-Titchmarsh theorem for multiplicative functions, J. reine angew. Math. 313 (1980), $161-170$. 